\title{
Aspect sensitive E- and F-region SPEAR-enhanced incoherent backscatter observed by the EISCAT Svalbard radar
}

\author{
R. S. Dhillon, T. R. Robinson, and T. K. Yeoman \\ Department of Physics and Astronomy, University of Leicester, University Road, Leicester LE1 7RH, UK \\ Received: 3 January 2008 - Revised: 26 November 2008 - Accepted: 26 November 2008 - Published: 6 January 2009
}

\begin{abstract}
Previous studies of the aspect sensitivity of heaterenhanced incoherent radar backscatter in the high-latitude ionosphere have demonstrated the directional dependence of incoherent scatter signatures corresponding to artificially excited electrostatic waves, together with consistent fieldaligned signatures that may be related to the presence of artificial field-aligned irregularities. These earlier high-latitude results have provided motivation for repeating the investigation in the different geophysical conditions that obtain in the polar cap ionosphere. The Space Plasma Exploration by Active Radar (SPEAR) facility is located within the polar cap and has provided observations of RF-enhanced ion and plasma line spectra recorded by the EISCAT Svalbard UHF incoherent scatter radar system (ESR), which is collocated with SPEAR. In this paper, we present observations of aspect sensitive E- and F-region SPEAR-induced ion and plasma line enhancements that indicate excitation of both the purely growing mode and the parametric decay instability, together with sporadic E-layer results that may indicate the presence of cavitons. We note consistent enhancements from fieldaligned, vertical and also from $5^{\circ}$ south of field-aligned. We attribute the prevalence of vertical scatter to the importance of the Spitze region, and of that from field-aligned to possible wave/irregularity coupling.
\end{abstract}

Keywords. Ionosphere (Active experiments; Plasma waves and instabilities; Polar ionosphere)

\section{Introduction}

Among the most important phenomena associated with overdense RF heating are the stimulation of non-propagating plasma density irregularities at the upper-hybrid height and

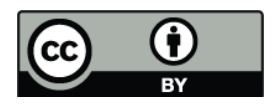

Correspondence to: R. S. Dhillon (ranvir.dhillon@ion.le.ac.uk) the excitation of Langmuir and ion-acoustic waves at the Omode reflection height (e.g. Robinson, 1989; Rietveld et al., 1993; Kohl et al., 1993; Mishin et al., 2004). These ionacoustic and Langmuir waves can be detected in the interaction region by radars pointing in a wide range of directions, including those close to the geomagnetic field direction (e.g. Stubbe et al., 1992; Kohl et al., 1993; Stubbe, 1996; Honary et al., 1999; Rietveld et al., 2000; Dhillon and Robinson, 2005). These wave modes give rise to enhancements in ion and plasma line incoherent scatter spectra and are thought to be caused by excitation of instabilities (Perkins and Kaw, 1971) that include the purely growing mode (PGM) (Fejer and Leer, 1972), also called the oscillating two-stream instability or modulational instability (Rietveld et al., 2002), and the parametric decay instability (PDI) (Fejer, 1979). These two instabilities are thought to be driven by the effects of the ponderomotive force (e.g. Kohl et al., 1993), thereby resulting in RF-induced Langmuir and ion-acoustic waves (PDI), and Langmuir waves associated with a stationary electron density perturbation (PGM). The PDI and PGM were first observed using the HF heating facility at Arecibo (Carlson et al., 1972; Gordon and Carlson, 1974) as summarized in the review by Carlson and Duncan (1977). As well as Fregion spectral enhancements, RF-induced features have also been noted in E-region data, including those from sporadic Elayers (e.g. Gordon and Carlson, 1976; Djuth, 1984; Schlegel et al., 1987; Djuth and Gonzales, 1988; Rietveld et al., 2002; Dhillon et al., 2007).

The SPEAR system (a relatively recent addition to the global array of HF high-power facilities) began experimental operations in April 2004, since when a wide variety of RF-induced effects produced using the high-power beam have been detected. These include SPEAR-induced plasma wave excitation and enhancements in field-parallel and fieldperpendicular scatter (Robinson et al., 2006; Scoffield et al., 2006; Yeoman et al., 2007, 2008; Dhillon et al., 2007; Clausen et al., 2008). Field-parallel SPEAR-induced spectral

Published by Copernicus Publications on behalf of the European Geosciences Union. 
modifications whose characteristics are consistent with PGM and PDI excitation, together with other plasma physical phenomena such as the effects of cavitons (Djuth and Gonzales, 1988), have been observed by the EISCAT Svalbard UHF incoherent scatter radar system (Robinson et al., 2006; Dhillon et al., 2007). In this paper, we add to these results by concentrating mainly upon RF-induced spectral enhancements and restrict our attention to E- and F-region SPEARenhanced spectral data accumulated by the EISCAT Svalbard Radar (ESR) during experimental SPEAR/ESR/CUTLASS campaigns conducted in December 2005, October 2006 and August 2007.

It is clear that the response of the magnetized ionospheric plasma to a high-power pump wave exhibits great complexity and variability depending upon the particular direction, relative to the geomagnetic field, in which the observations of RF-induced phenomena are made. This dependence, or "aspect sensitivity", motivates us to investigate the underlying plasma physical phenomena that give rise to such directional variability. As noted by Robinson et al. (2006), incoherent radar backscatter data display less aspect sensitivity than data collected using coherent backscatter radars, which rely on orthogonal backscatter from field-aligned irregularities and therefore provide backscatter powers that maximize in directions perpendicular to the geomagnetic field. Previous studies involving the aspect sensitivity of RF-induced ionospheric responses have included those in which significant electron temperature enhancements have been noted (e.g. Rietveld et al., 2003), incoherent scatter radar observations of ion and plasma line enhancements, together with their altitude characteristics (e.g. Djuth et al., 1994; Isham et al., 1999; Dhillon and Robinson, 2005), measurements of artificial airglow (e.g. Kosch et al., 2000; Pedersen et al., 2003; Kosch et al., 2004) and observations of stimulated electromagnetic emissions (Isham et al., 2005). These previous investigations highlighted the importance of many directions relative to the geomagnetic field and the study presented here again provides evidence of the apparent special nature of certain directions, including field-aligned.

The experimental configuration and certain technical aspects of the SPEAR and ESR systems are described in Sect. 2 of this paper, with Sect. 3 providing an overview of the experiments conducted in the SPEAR campaigns. A discussion of the results is presented in Sect. 4 and a summary of conclusions is given in Sect. 5 .

\section{Instrumentation}

SPEAR is a relatively new combined HF high-power (heater) and coherent scatter radar system, first described by Wright et al. (2000), located in the vicinity of Longyearbyen (Spitzbergen in the Svalbard archipelago with a magnetic dip angle of approximately $8.5^{\circ}$ ) and is designed to carry out a range of space plasma investigations of the polar iono- sphere and magnetosphere. A comprehensive description of the SPEAR system, including scientific goals, capabilities and initial results, together with technical specifications and operating constraints, has been presented by Robinson et al. (2006). During the intervals covered here, SPEAR operated using the full $6 \times 4$ array, transmitting O-mode-polarized radio waves in the field-aligned direction with a frequency of $4.45 \mathrm{MHz}$ and an effective radiated power (ERP) of approximately $15 \mathrm{MW}$. The half-power beam width is approximately $21^{\circ}$.

The CUTLASS coherent scatter radars (Milan et al., 1997) were also in operation during the experimental intervals presented below. Both the Iceland and Finland radars ran an experimental mode that used $15 \mathrm{~km}$ range gates on channel A and $45 \mathrm{~km}$ range gates on channel B, with beam 6 of Iceland and beam 9 of Finland overlooking the SPEAR site. Because of a combination of the frequency sweeps $(11-13 \mathrm{MHz})$, integration time (1 s) and beam sweeps (beams 3-5) that were used, the temporal resolution of the data from any specific range-beam cell varied from 9-15 s. Further details of the CUTLASS operating modes utilized during the experiments are given by Robinson et al. (2006).

The incoherent scatter radar data presented below were recorded using the ESR (Wannberg et al., 1997), which is collocated with SPEAR. The ESR, which operates at frequencies close to $500 \mathrm{MHz}$, was used to detect the Langmuir and ion-acoustic waves generated during the excitation of instabilities near the O-mode reflection height. During all three campaigns from which data are presented, i.e. those conducted during December 2005, October 2006 and August 2007, the ESR ran an experimental mode that used the steerable $32 \mathrm{~m}$ dish, whose pointing direction will be discussed in more detail below, to collect both ion and plasma line data using long pulses. The ion line spectra were obtained on two channels, with transmitter frequencies of 499.9 and $500.3 \mathrm{MHz}$, using $350 \mu$ s long pulses with a sampling rate of $10 \mu \mathrm{s}$. The height-discriminated ion line spectral data for both channels were obtained for an altitude range of 86$481 \mathrm{~km}$ with a resolution of about $28 \mathrm{~km}$. The frequency resolutions of data from the two channels were $2.0 \mathrm{kHz}$ with the frequency ranges of both being $\pm 50 \mathrm{kHz}$. Only data from the $499.9 \mathrm{MHz}$ channel have been presented in this paper. The plasma line data were recorded on a separate channel, with a transmitter frequency of $500.1 \mathrm{MHz}$ and a resolution of $13.0 \mathrm{kHz}$, using a $1500 \mu$ s long pulse with a sampling rate of $0.6 \mu \mathrm{s}$. During the first two data intervals covered in this paper, plasma line spectra were obtained in two overlapping frequency bands of $3.2-4.8$ and $4.5-6.1 \mathrm{MHz}$ both upshifted and downshifted from the transmitter frequency. For the third interval, frequency bands upshifted and downshifted by $3.65-5.25 \mathrm{MHz}$ (centred on the SPEAR frequency of $4.45 \mathrm{MHz}$ ) and $4.5-6.1 \mathrm{MHz}$ were used. The plasma line data were obtained from altitude ranges of 90-315 and 240$465 \mathrm{~km}$, although only data from the $90-315 \mathrm{~km}$ altitude range are presented in this paper. Because of the long pulses 

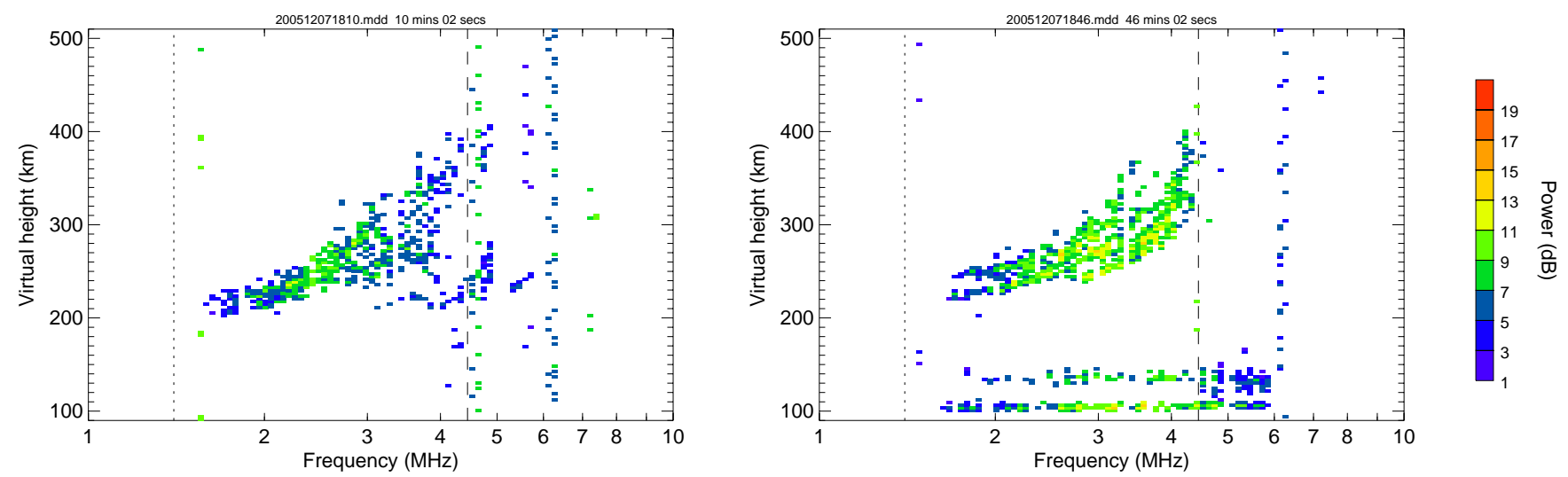

Fig. 1. Ionograms taken at 18:10 and 18:46 UT on 7 December 2005 (interval 1). The ionogram from 18:46 UT has signatures consistent with a porous sporadic E-layer that affects waves with frequencies from $2-6 \mathrm{MHz}$. There is also evidence of second-hop scatter over this frequency range. These ionograms, together with those shown in Figs. 2 and 3, were recorded using the Svalbard ionosonde during dropouts when SPEAR was not transmitting. In these figures, the SPEAR frequency of $4.45 \mathrm{MHz}$ is denoted using a vertical dashed line.

used, together with a lack of data regarding the echo strength versus range across the long pulse, the plasma line data are not discriminated in altitude. The temporal resolution of both the ion and the plasma line data was $5.0 \mathrm{~s}$.

In order to investigate the aspect sensitivity of SPEARenhanced ESR backscatter, the steerable $32 \mathrm{~m}$ diameter dish was oriented in several directions in the magnetic meridian plane, i.e. its azimuth was fixed (with a geographic bearing of $182.1^{\circ}$ ) while its elevation varied according to predetermined cycles, all centred on field-aligned (FA, with a geographic azimuth and elevation of $182.1^{\circ}$ and $81.6^{\circ}$ respectively). A seven-direction cycle, with a dwell-time of two minutes per direction, was applied during the December 2005 and October 2006 campaigns. This cycle used a separation of $5^{\circ}$ between adjacent pointing directions, thereby resulting in an angular extent of $30^{\circ}$ centred on field-aligned. Using the convention that angular displacements in the direction of increasing elevation angle, from field-aligned, will be labelled as positive, and angular displacements in the direction of decreasing elevation angle will be labelled as negative, we have denoted these seven pointing directions as follows: FA+15, FA+10, FA+5, FA, FA-5, FA-10 and FA-15. A fifteen-direction cycle with a higher spatial resolution of $2^{\circ}$ between adjacent pointing directions was used during the August 2007 campaign. This resulted in an angular extent of $28^{\circ}$ centred on field-aligned. Adopting the same convention for labelling pointing directions gives directions from $F A+14$ to $F A-14$, via FA+12, FA+10...FA etc. Also, a dwell-time of one minute per direction was used for this higher-resolution cycle.

\section{E- and F-region SPEAR-enhanced incoherent scatter data}

This section covers aspect sensitive E- and F-region ESR observations of SPEAR-enhanced ion and plasma line spectral data collected during campaigns conducted in December 2005, October 2006 and August 2007. During all three of the intervals discussed below SPEAR continuously transmitted O-mode-polarized radiation, at $4.45 \mathrm{MHz}$, while pointing in the field-aligned direction. The three data intervals covered here are 18:10-20:10 UT on 7 December 2005 (interval 1), 11:00-16:00 UT on 9 October 2006 (interval 2) and 14:10-16:00 UT on 15 August 2007 (interval 3). As described in Sect. 2, a seven-direction ESR scanning cycle was adopted during intervals 1 and 2, whereas a fifteendirection cycle was applied during interval 3. The SPEARenhanced spectra from these intervals illustrate clearly the effects of an HF high-power pump wave in (a) an irregular ionosphere with traces of a sporadic E-layer (interval 1), (b) an ionosphere with a temporally varying F-region with many features (interval 2) and (c) an ionosphere with a fairly weak F-region (interval 3). These ionospheric conditions were deduced using ionograms, recorded using the Svalbard ionosonde (Robinson et al., 2006), which were taken during each of these data intervals. Although SPEAR was ostensibly transmitting continuously, there were periods during which transmissions did not occur. These dropouts were caused by a number of factors, including activation of the Longyearbyen airport interlock, which inhibits transmissions from SPEAR and the ESR during the presence of aircraft, operational and technical difficulties, and compulsory interruption of our observations in order to collect data in support of the International Polar Year (IPY), with such experiments running well into 2008. Because of inability to operate the Svalbard ionosonde simultaneously with SPEAR when it is 

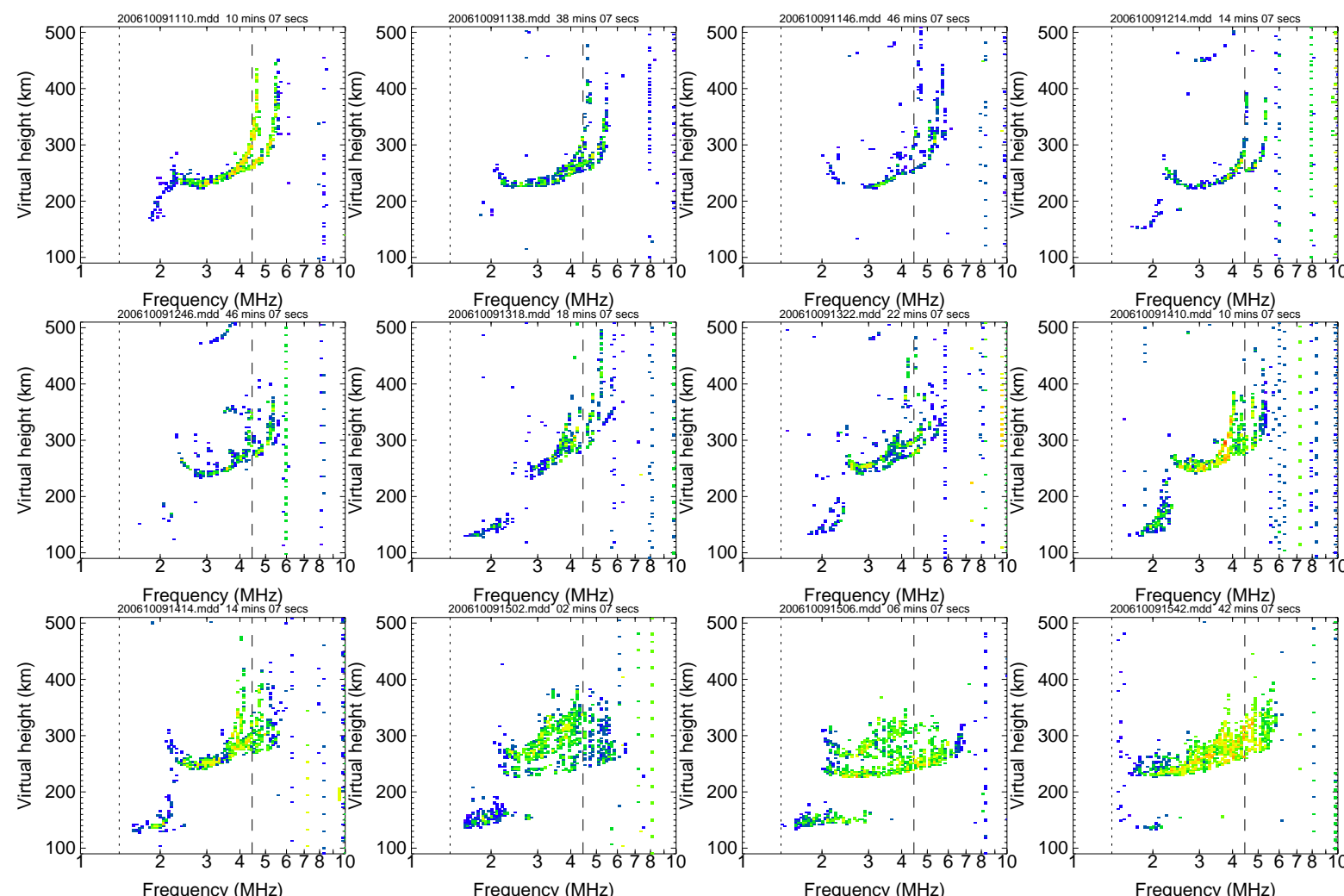

Fig. 2. A series of ionograms from 11:10 to 15:42 UT on 9 October 2006 (interval 2). These ionograms show an ionosphere with an F-region that evolves over time, gradually becoming less structured and more disturbed.

functioning in high-power mode, real-time ionograms created within the nominal SPEAR-on interval could only be obtained during these dropouts. These ionograms, together with others from immediately before and immediately after SPEAR operation, are shown in Figs. 1, 2 and 3. Figure 1 shows ionograms taken at 18:10 and 18:46 UT on 7 December 2005 (i.e. just before and during interval 1). The ionogram taken at 18:46 UT has signatures consistent with a porous sporadic E-layer (e.g. Kagan et al., 2000; 2002) that affects waves with frequencies from $2-6 \mathrm{MHz}$. There is also evidence of second-hop scatter over this frequency range. Figure 2 shows a series of ionograms, from 11:10 to 15:42 UT on 9 October 2006 (within interval 2), recorded before, within and after the duration in which SPEAR was nominally transmitting. These ionograms show an ionosphere with an F-region that evolves over time, gradually becoming less structured and more disturbed over time. Figure 3 shows another series of ionograms, in this case beginning at 14:06 UT and proceeding to 16:02 UT on 15 August 2007 (i.e. through interval 3). These ionograms are suggestive of an irregular ionosphere, which at least for a period during the interval is underdense. There is also evidence of an E-layer that interacts with radio waves having frequencies from about 2 to $4 \mathrm{MHz}$, and possibly higher. The vertical dashed line be- tween 4 and $5 \mathrm{MHz}$ in all of these ionograms corresponds to the SPEAR frequency of $4.45 \mathrm{MHz}$. Although the altitude resolution in the ionograms is $6 \mathrm{~km}$, signatures with extended altitude ranges are sometimes seen and these may be caused by the oblique nature of some of the echoes detected by the ionosonde receiver/processing system.

We turn now to the ESR incoherent scatter radar data that were collected during the three intervals. The altitudinal and directional dependence of the ion line spectra will be discussed later, with the data shown in Figs. 7 to 10, but we now consider overviews of the ESR ion and plasma line data. We have shown data from intervals 1 and 3 but have chosen not to illustrate those from interval 2 , because showing the spectral amplitudes over the entire five-hour interval would obscure the multi-directional enhancements that we wish to highlight. The overviews are illustrated using Figs. 4 and 5 , which contain panels that show time series of the central ion line (CIL) and upshifted (UPL) and downshifted (DPL) plasma line amplitudes. The central ion line corresponds to the central spectral minimum, which is at the ion line radar frequency $(499.9 \mathrm{MHz})$. We have not shown the amplitudes of the (F-region) upshifted and downshifted ion lines, which correspond to the ion-acoustic peaks, as SPEAR-induced enhancements in these spectral components have tended to 

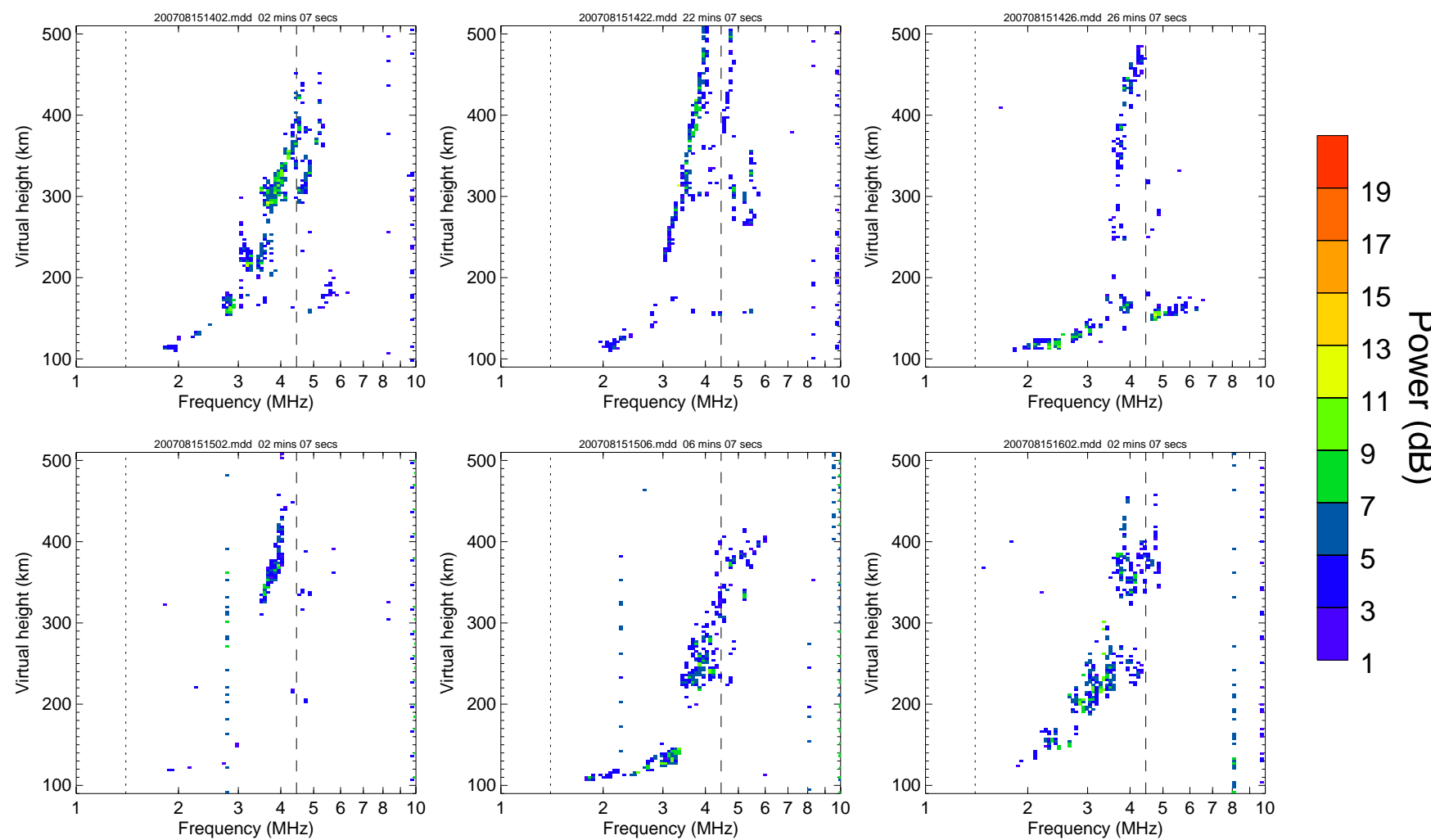

Fig. 3. Another series of ionograms beginning at 14:06 UT and proceeding to 16:02 UT on 15 August 2007 (interval 3). The features in these ionograms are consistent with an irregular ionosphere, which at least for a period during the interval is underdense. There is also evidence of an E-layer that interacts with radio waves having frequencies of about $2-4 \mathrm{MHz}$.

occur simultaneously with enhancements in the central ion line (e.g. Robinson et al., 2006). Also, the upshifted and downshifted plasma lines are at $\pm 4.45 \mathrm{MHz}$ from the plasma line radar frequency $(500.1 \mathrm{MHz})$. In both figures, the startand end-times of the ESR scanning cycles are identified using vertical light blue lines. The periods during which the ESR pointed field-aligned are denoted using black rectangles at the top and bottom of the data panels, with each scanning cycle being labelled by the circled numbers located above the panels. The red bars, of varying thickness, indicate periods of high ion or plasma line amplitude consistent with the action of SPEAR.

Figure 4 shows ion and plasma line data from interval 1 ( 7 December 2005, 18:10-20:10 UT), where seven cycles were used, with the pointing directions in a given cycle proceeding from FA+15 to FA-15. The ionogram from 18:46 UT shown in Fig. 1 was collected during activation of the airport interlock. Panels (a) to (d) show F-region (150-250 km) CIL, E-region $(80-110 \mathrm{~km})$ CIL, UPL and DPL data respectively. These altitude ranges were chosen for the E- and F-regions by virtue of the fact that the principal spectral enhancements occurred within them (see Figs. 7 to 10). Panel (e) shows the angular displacement of the $32 \mathrm{~m}$ dish in the magnetic meridian plane (azimuth fixed at $182.1^{\circ}$ ). Although this dis- placement can have values that exceed $90^{\circ}$, it has been labelled as the elevation angle, to which it is identical for values from $0^{\circ}$ (horizontal) to $90^{\circ}$ (vertical). We shall examine the data by considering significant signatures in the plasma line and/or ion line data and study the directional dependence of the apparent SPEAR-induced responses. Such SPEAR-induced spectral enhancements are easier to identify in the plasma line data, as significant increases in the UPL and DPL amplitudes (at $\pm 4.45 \mathrm{MHz}$ from the radar frequency) will most probably be caused by SPEAR-excited instabilities. This provides us with a reliable method by which to distinguish between natural and artificial features and we have positioned the red bars in this figure so as to highlight the plasma line enhancements. By inspection, we notice clear multi-directional SPEAR-induced spectral enhancements that occur during scanning cycles 2, 4 and 6, and we shall concentrate on these. Considering cycle 2 first, we see notable plasma line enhancements, clear in panels (c) and (d), when the ESR pointed in FA+15, FA+10 and FA-5. The E-region ion line enhancement in FA, seen in panel (b), is not accompanied by an increase in the plasma line amplitude and this absence may cast doubt upon whether this ion line amplitude increase is a SPEAR-induced effect. Turning next to cycle 4, we find clear plasma line and E-region 


\section{ESR BACKSCATTER DATA}

F-CIL, E-CIL, UPL and DPL on 07/12/2005

(a)

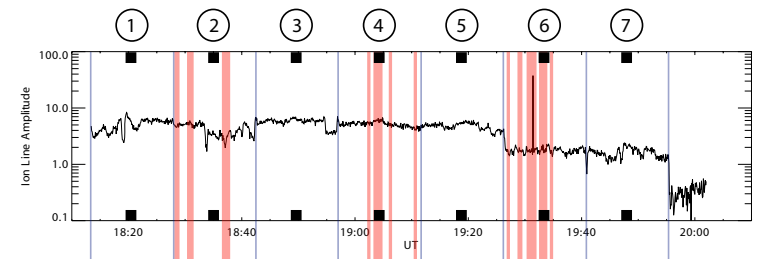

(b)

(c)

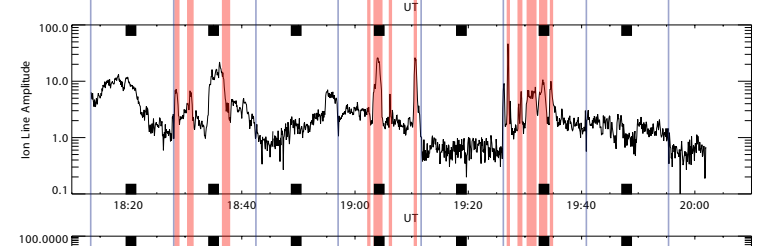

(d)
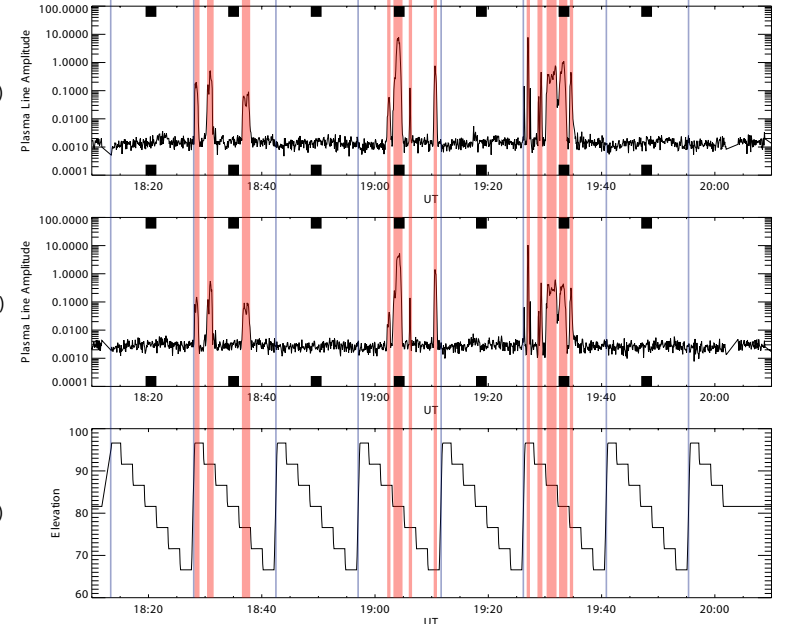

Fig. 4. Ion and plasma line amplitudes from 7 December 2005 (interval 1). Panels (a) to (d) show the F-region central ion line $(150-250 \mathrm{~km})$, E-region central ion line $(80-110 \mathrm{~km})$, the upshifted plasma line and the downshifted plasma line respectively. Panel (e) shows the elevation angle (angular displacement from horizontal) of the $32 \mathrm{~m}$ dish. In this figure, and in Fig. 5, the start- and end-times of the ESR scanning cycles are identified using vertical light blue lines. The periods during which the ESR pointed fieldaligned are denoted using black rectangles at the top and bottom of the data panels, with each scanning cycle being labelled by the circled numbers located above the panels. The red bars, of varying thickness, indicate periods of high ion or plasma line amplitude consistent with the action of SPEAR. There are clear ion and plasma line spectral enhancements from several directions, including from $\mathrm{FA}+15, \mathrm{FA}+10, \mathrm{FA}+5, \mathrm{FA}, \mathrm{FA}-5$ and FA-15, with notable enhancement occurring in FA. The upshifted and downshifted plasma line enhancements occur mainly in the E-region and are clearly well correlated, both with each other and with the ion line enhancements.

ion line enhancements in FA+5, FA, FA-5 and FA-15, with the strongest plasma line enhancement occurring in FA. Cycle 6 has enhancements in the five directions from $F A+15$ to FA-5 and these again occur in the plasma line and Eregion ion line data. Additionally, there is a possible E-region ion line enhancement in FA-15 in cycle 3. Also, there are

\section{ESR BACKSCATTER DATA}

F-CIL, E-CIL, UPL and DPL on 15/08/2007

(a)

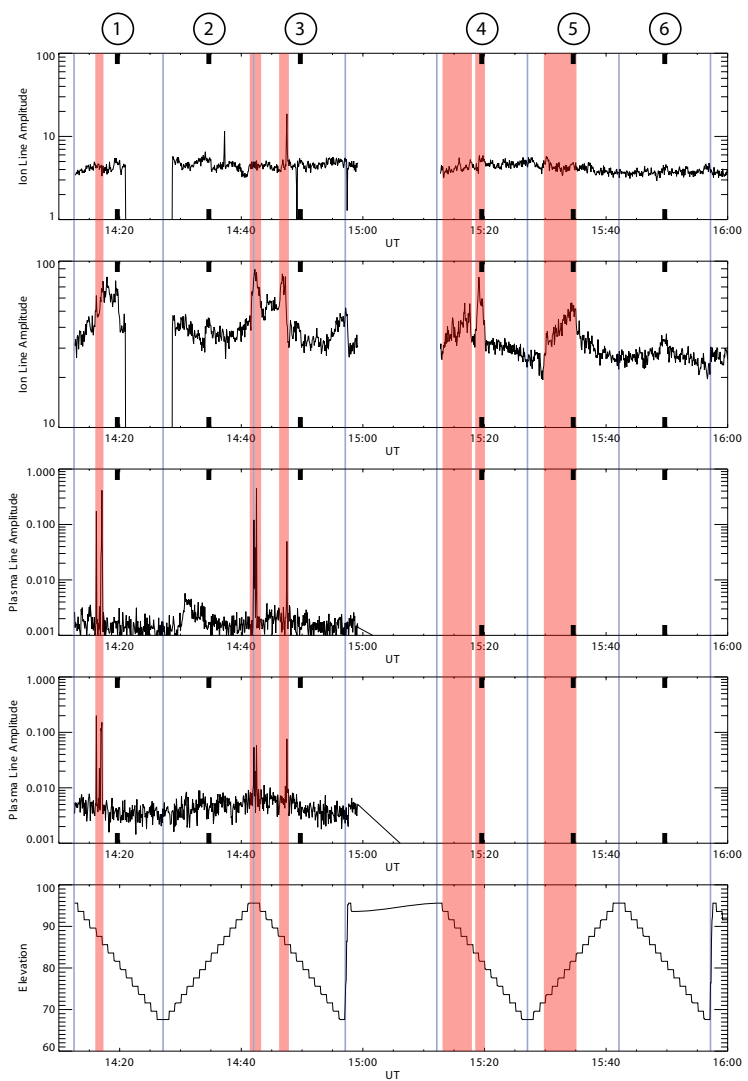

Fig. 5. Ion and plasma line amplitudes from 15 August 2007 (interval 3). Panels (a) to (d) show F-region central ion line, E-region central ion line, and the upshifted and downshifted plasma line respectively. Panel (e) again shows the elevation angle (angular displacement) of the $32 \mathrm{~m}$ dish. The demarcation of the scanning cycles and the labelling of field-aligned are as for Fig. 4, with the red bars again highlighting the spectral enhancements. Definite enhancements were seen in pointing directions from $\mathrm{FA}+14$ to $\mathrm{FA}+4$ and in FA. As for data from interval 1, shown in Fig. 4, these enhancements occurred mainly in the E-region.

few signs of SPEAR-induced spectral enhancements in the F-region ion line amplitude, shown in panel (a). We therefore conclude that the bulk of the enhancements occur in the E-region. Clearly, the E-region CIL amplitude varied appreciably during the SPEAR-on periods and this is accompanied by considerable variability in the F-region CIL amplitude. The SPEAR-induced UPL and DPL enhancements are clearly well correlated, both with each other and with the Eregion ion line enhancements.

We now comment briefly on data from interval 2 ( 9 October 2006, 11:00-16:00 UT), where sixteen scanning cycles were used, with the pointing directions in a given cycle again proceeding from FA+15 to FA-15. Due to plasma line hardware problems, the only useful measurements collected 
during this interval were the ion line data. Because of the natural variability in the plasma density of the polar cap ionosphere over SPEAR, and hence in the associated ion line spectral amplitude (e.g. Robinson et al., 2006; Dhillon et al., 2007), unambiguously identifying SPEAR-induced features became more difficult and we attempted to discern such effects by inspection. We were aided by recognizing the consistent (apparent over several cycles) ion line enhancements in FA. Also, we note that these enhancements, which occurred mainly in the F-region and which were similar to SPEAR-induced F-region enhancements reported previously by Robinson et al. (2006) and Dhillon et al. (2007), were not as obvious for pointing directions away from FA. Despite the difficulty in distinguishing between natural and artificial signatures, we were able to identify scanning cycles containing evidence of SPEAR-enhanced multi-directional enhancements. These scanning cycles are discussed below and the corresponding ion line spectra are shown in Figs. 8 and 9.

Figure 5 shows ion and plasma line data from interval 3 (15 August 2007, 14:10-16:00 UT). For this interval the high-resolution ( $2^{\circ}$ angular separation) scanning mode was utilized and six scanning cycles were applied. Also, the scanning sequence was reversed in the second and fifth cycles, i.e. going from FA-14 to FA+14 instead of FA+14 to FA-14, which was the sequence used in the other cycles. The panel layout in this figure is the same as that used in Fig. 4, with panels (a) to (d) showing F-region CIL, E-region CIL, UPL and DPL respectively. Panel (e) again shows the elevation angle (angular displacement) of the $32 \mathrm{~m}$ dish. Plasma line hardware problems were again present and these data were only available for 14:10-15:00 UT. As for interval 1, we have used these plasma line data to identify periods of multidirectional enhancement, which have been highlighted using red bars. From 15:10-16:00 UT, when plasma line data were absent, we tried to identify SPEAR-induced ion line spectral enhancements by inspection. The loss of data from approximately 14:20-14:30 UT resulted from the airport interlock, whereas that from 15:00-15:10 UT was caused by mandatory suspension of our experiment in order to accommodate data collection for the IPY experiment. Definite E-region ion line amplitude increases were seen in FA for cycles 2, 4 and 6. There are additional increases in FA+8 and FA+6, i.e. close to vertical, in cycle 1 . There are also definite E-region ion and plasma line spectral enhancements at the end of cycle 2 and beginning of cycle 3 in FA+14. Cycle 3 also contains ion and plasma line enhancements in FA+4 and FA+6. In cycle 4, E-region ion line amplitude increases are again present for directions from $\mathrm{FA}+4$ to $\mathrm{FA}+12$. The higher ion line amplitudes after about 15:30 UT in cycle 5 for all directions from FA-10 to FA may also be SPEAR-induced. As for data from interval 1 most of these enhancements occurred in the E-region CIL data.

The CUTLASS Finland and Iceland radars were operating during the three data intervals and they collected data using the scanning mode that was described in Sect. 2. SPEAR-

\section{SUPERDARN PARAMETER PLOT}

CUTLASS Finland and Iceland Backscatter Power on 9 Oct 2006

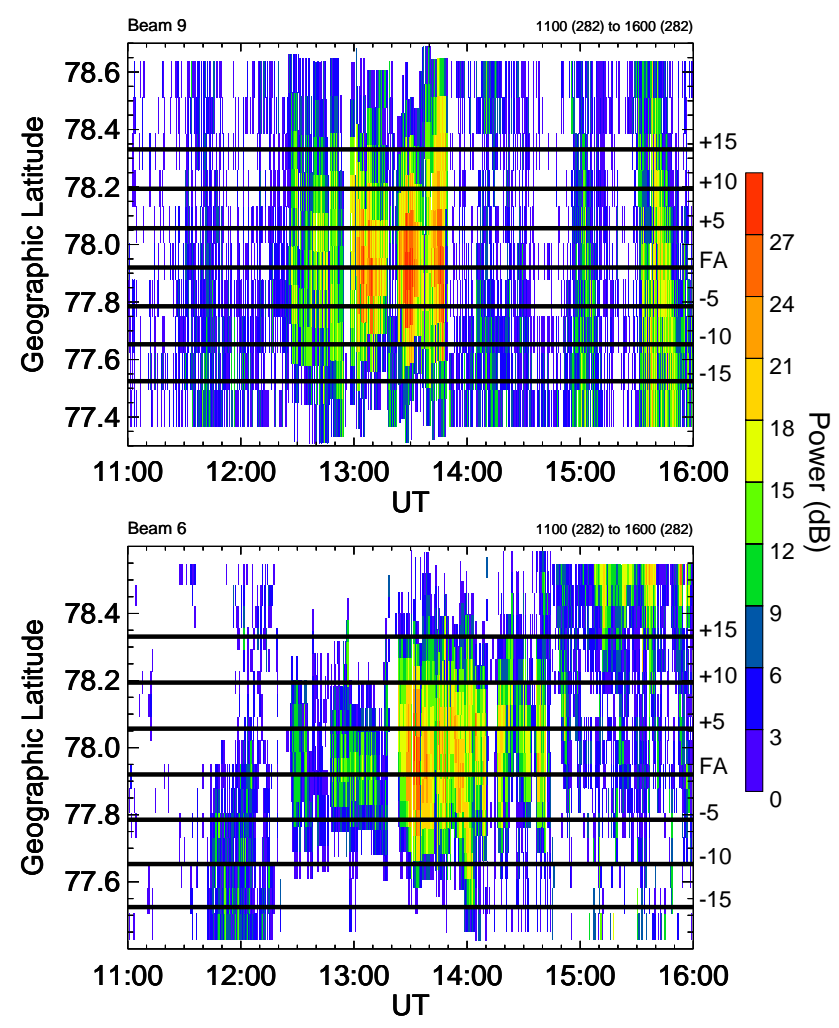

Fig. 6. A range-time-intensity plot of the repositioned CUTLASS backscatter power from 9 October 2006 (interval 2). The upper and lower panels show data from Finland Channel A (beam 9) and Iceland Channel A (beam 6) respectively. The horizontal black bars in each panel show the overlaid geographic latitudes, calculated using a typical interaction altitude of $180 \mathrm{~km}$, corresponding to the seven ESR pointing directions. The SPEAR beam pointed field-aligned and this corresponds to the highest backscatter power, with appreciably lower powers for pointing directions furthest from FA, i.e. $\mathrm{FA} \pm 10$ and $\mathrm{FA} \pm 15$.

enhanced CUTLASS backscatter was seen only during interval 2, which, together with the F-region ion line enhancements and ionograms, is consistent with SPEAR interacting with the plasma in the F-region. Because of the $1 \frac{1}{2} 2$-hop propagation path via which the CUTLASS rays travel to and from the ionosphere over the SPEAR facility (Yeoman et al., 2007,2008 ) it is found that the calculated position of the scatter is highly susceptible to changes in the ionospheric plasma density. This results in apparent migration of the patch of artificial backscatter over time. In order to be able to study SPEAR-enhanced coherent backscatter properly over extended temporal periods, and investigate its effects on the aspect sensitivity of SPEAR-enhanced incoherent backscatter, this apparent motion must be removed. This has been done by assuming that the SPEAR-enhanced coherent backscatter 


\section{ESR BACKSCATTER DATA}

Ion Line Spectra (scanning cycles 2, 4 and 6) on 07/12/2005
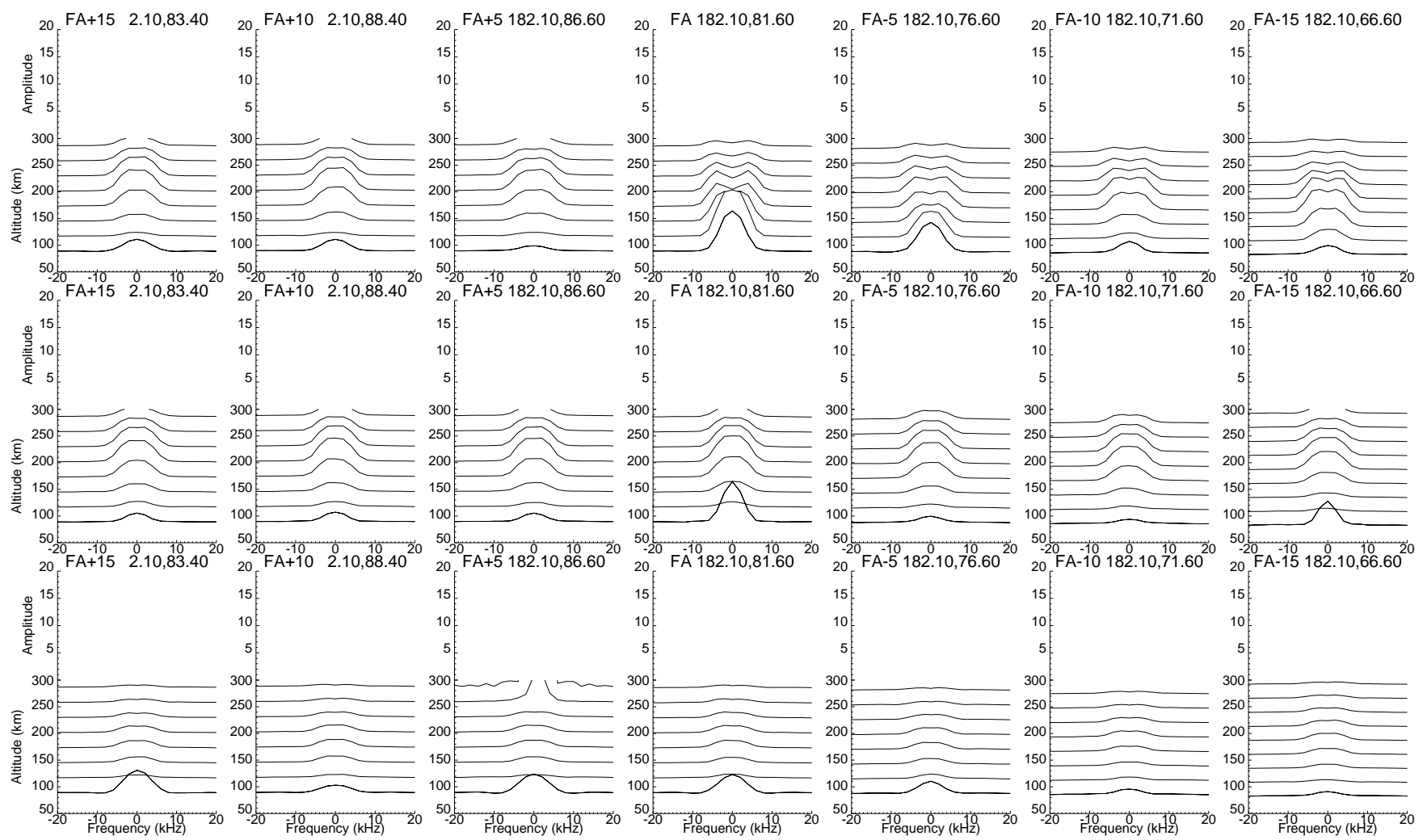

Fig. 7. Ion line spectra for scanning cycles 2, 4 and 6 from interval 1. For these data, and those from interval 2 shown in Figs. 8 and 9 , each of the seven panels in each row shows temporally averaged ion line spectra from all times during which the ESR pointed in a specific direction, which ranged from FA+15 to FA-15. Each panel direction is labelled using its designation, e.g. FA+10, followed by the azimuth and elevation of that pointing direction. The horizontal axis in each data panel shows the frequency and the other two axes show the altitude and the spectral amplitude. It is clear that significant E-region enhancements occur in FA+15, FA+5, FA and FA-5, with notable increases in FA. Also, the central parts of the F-region spectra in FA+15, FA+10 and FA+5 are clearly enhanced.

with the highest amplitude at a given time corresponds to the most intense part of the SPEAR beam, which in this case was pointing in the field-aligned direction. Since the location of SPEAR is known $\left(78.15^{\circ} \mathrm{N}, 16.05^{\circ} \mathrm{E}\right.$ geographic), this technique may be used to reposition the scatter such that its apparent movement over time can be eliminated. A further essential requirement involves confirming that the manipulated scatter was genuinely SPEAR-induced, and not naturally occurring scatter that happened to be in the vicinity. This was done by examining the spectral widths of the backscatter. Since artificial backscatter generally has lower spectral widths than those of natural backscatter (e.g. Dhillon et al., 2002), this spectral characteristic may be used to differentiate SPEAR-enhanced CUTLASS backscatter from natural backscatter. By applying this method to the data from this interval, we were able to identify the artificial backscatter with some confidence. These corrective methods have been applied to CUTLASS data taken during in- terval 2 and a range-time-intensity plot of the repositioned artificial backscatter, for 11:00-16:00 UT, is shown in Fig. 6. The upper and lower panels of this figure correspond to data from Finland Channel A (beam 9) and Iceland Channel A (beam 6) respectively. The labelled horizontal black bars in each panel show the overlaid geographic latitudes of the seven ESR pointing directions. These were calculated for a typical interaction altitude during the interval of $180 \mathrm{~km}$. The variation in the backscatter amplitude across the modified patch, as seen by both radars, results from the highest backscatter power corresponding to the centre of the SPEAR beam. The temporal variability in the width of the backscatter patch may result, at least in part, from the intrinsic nature of the polar cap ionosphere, through which patches of overdense plasma frequently travel (Robinson et al., 2006; Dhillon et al., 2007). The variability in the backscatter amplitude, at any spatial position, may also be explained by including the effects of changing ionospheric propagation. It is 


\section{ESR BACKSCATTER DATA}

\section{Ion Line Spectra (scanning cycles 1, 4 and 5) on 09/10/2006}
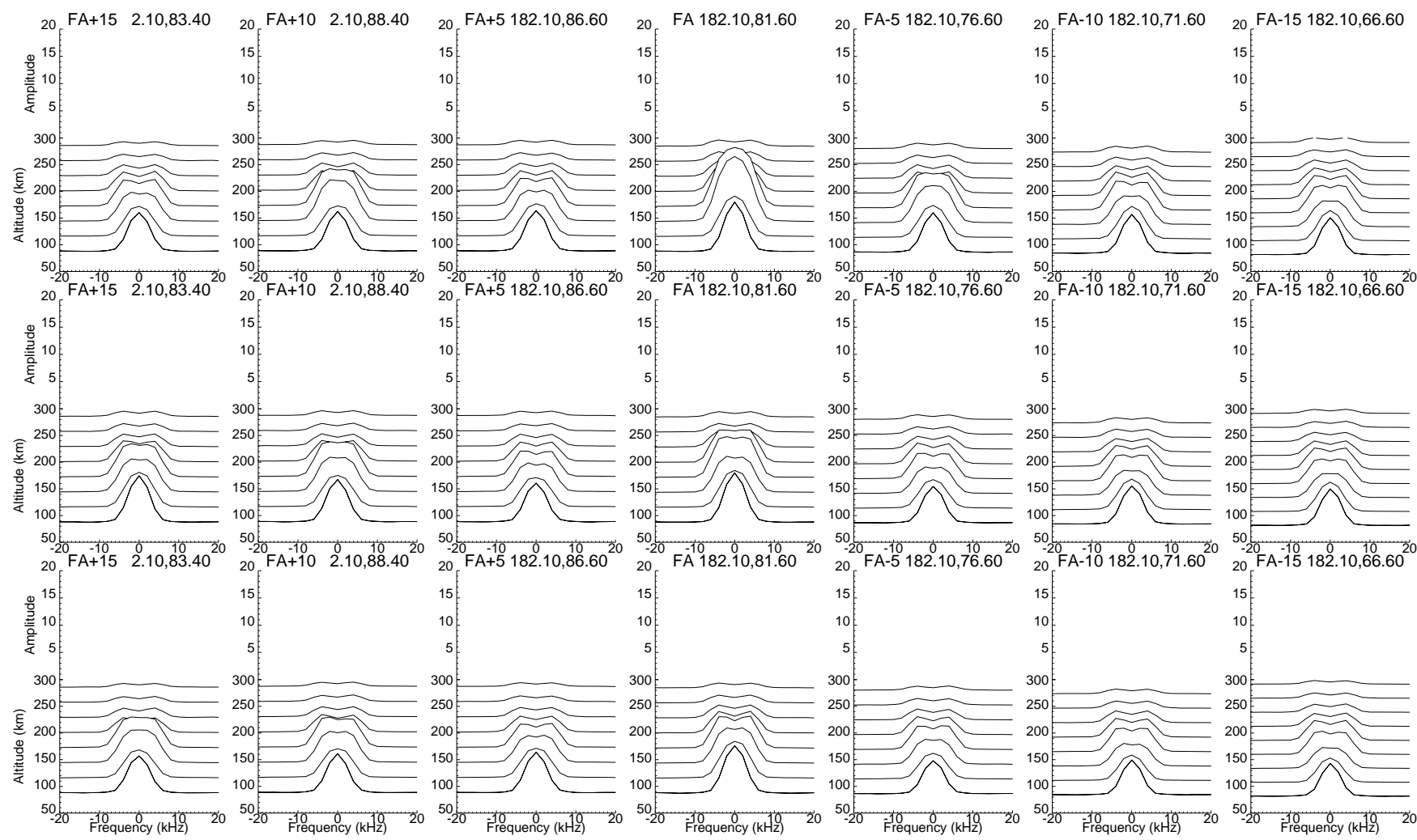

Fig. 8. Ion line spectra from scanning cycles 1, 4 and 5 from interval 2. The data are displayed using the same format and labelling as that applied in Fig. 7. The main features here are the probable SPEAR-induced F-region enhancements in FA+15, FA+10, FA and FA-5, with the highest amplitude occurring in FA. These data are generally characterized by increases in the amplitudes of the ion-acoustic peaks and of the central part of the spectrum. There is evidence of an E-region throughout the three cycles, but this appears to be unaffected by the SPEAR high-power wave.

notable that the powers have fallen appreciably for pointing directions furthest from FA, i.e. FA \pm 10 and $\mathrm{FA} \pm 15$. This variation in the backscatter amplitudes implies that the effects of field-aligned irregularities on ESR incoherent scatter data will most likely be seen in data from FA, with the influence of these irregularities decreasing with increasing aspect angle. This in turn is consistent with the aspect sensitivity mechanism proposed by Dhillon and Robinson (2005), whereby the field-aligned irregularities may interact with SPEAR-excited electrostatic waves. We shall return to these results later in the discussion given in Sect. 4.

Previously we discussed overviews of ion and plasma line data from intervals 1,2 and 3. We shall now examine the directional and altitudinal dependence of the ion line spectra from the three data intervals. Starting with interval 1 , we note that data taken during scanning cycles 2,4 and 6 exhibited significant multi-directional spectral enhancements. The corresponding ion line spectra are shown in Fig. 7, which has three rows of panels that show the data from cycles 2,4 and
6, respectively. Each of the seven panels in each row shows temporally averaged ion line spectra from all times during which the ESR pointed in a specific direction in that cycle and each pointing direction, from FA+15 to FA-15, is labelled using its designation, e.g. FA+10, followed by the azimuth and elevation of that pointing direction. The horizontal axis in each data panel shows the frequency and the other two axes show the altitude and the spectral amplitude. Taking cycle 2 first, it is clear that significant E-region enhancements occur in FA and FA-5, with the highest amplitude occurring in FA. The central parts of the F-region spectra in FA+15, $\mathrm{FA}+10$ and FA+5 are clearly enhanced, indicating the action of SPEAR (Robinson et al., 2006; Dhillon et al., 2007). The data from cycle 4 have similar strong E-region enhancements in FA, although in this case they are accompanied by F-region signatures that are present in spectra from all directions. These E-region characteristics are repeated in the data from cycle 6, with noticeable enhancements in FA+15, FA+5 and FA, although F-region signatures are not apparent. 


\section{ESR BACKSCATTER DATA}

\section{Ion Line Spectra (scanning cycles 7, 11 and 14) on 09/10/2006}
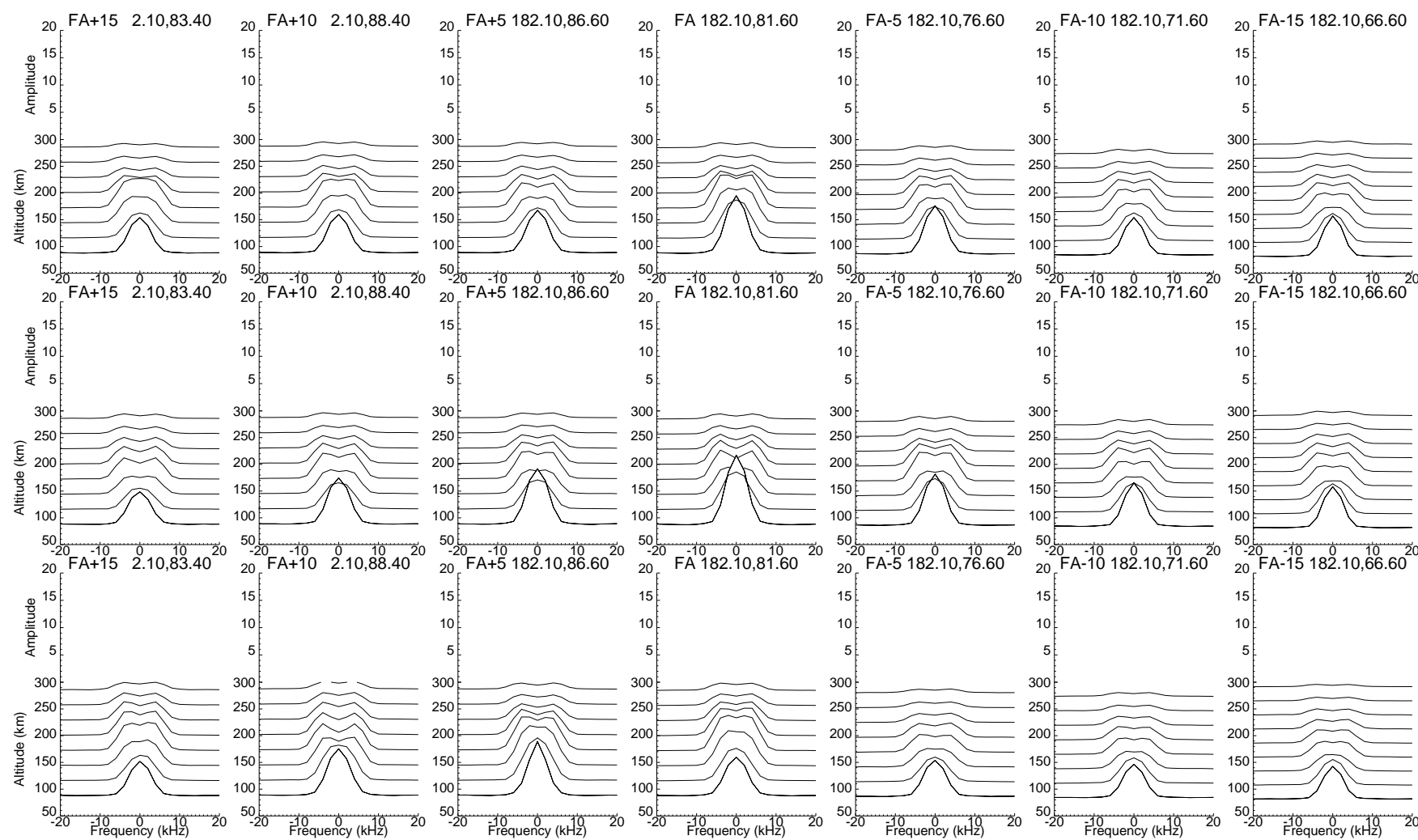

Fig. 9. Ion line spectra from scanning cycles 7, 11 and 14 from interval 2. The data are displayed using the same format and labelling as that applied in Fig. 7. These data contain fewer examples of F-region enhancements, although they appear to show enhancements in the E-region from $\mathrm{FA}+10, \mathrm{FA}+5, \mathrm{FA}$ and $\mathrm{FA}-5$.

The single central peaks present in these E-region spectra are indicative of collisional plasma and the increase in amplitude of these peaks during SPEAR-on is consistent with previous observations of RF-enhanced E-region ion line spectra (e.g. Rietveld et al., 2002; Dhillon et al., 2007). In the Fregion, SPEAR-induced spectral enhancements were found to occur within the altitude range of $150-250 \mathrm{~km}$. The Fregion ion line spectrum has its usual form, with two ionacoustic peaks whose separation is related to the plasma temperature. This ion-acoustic peak separation remained unchanged while SPEAR transmitted, which implies that there was a negligible change in the electron temperature during SPEAR-on. The symmetric nature of the spectra presented here is in agreement with the high degree of symmetry seen in previous SPEAR-enhanced F-region ion line spectra (Robinson et al., 2006), which were obtained when no significant E-region was present. It also contrasts with the asymmetric F-region spectral data, reported by Dhillon et al. (2007), which were obtained during the presence of a sporadic E-layer.
We turn now to interval 2, where, of the sixteen scanning cycles that were used, cycles $1,4,5,7,11$ and 14 contained possible multi-directional SPEAR-induced ion line enhancements. As we have noted above, the modified spectra, particularly in the F-region, are similar in form to previous SPEAR-enhanced ion line spectra (Robinson et al., 2006; Dhillon et al., 2007) and this similarity provides evidence that the spectral modifications are caused by the action of SPEAR. The data are shown in Figs. 8 and 9 with Fig. 8 covering data from scanning cycles 1,4 and 5, and Fig. 9 displaying data from cycles 7,11 and 14 . These data are presented in the same form as those in Fig. 7, with each row of panels corresponding to a particular scanning cycle and the panels, from left to right, denoting the pointing directions from FA+15 to FA-15. The three axes again show the frequency, the altitude and the spectral amplitude. The main features in Fig. 8 are the probable SPEAR-induced Fregion enhancements in FA+15, FA+10, FA and FA-5, with the highest amplitude occurring in FA. These data are generally characterized by increases in the amplitudes of the ion-acoustic peaks and of the central part of the spectrum. 


\section{ESR BACKSCATTER DATA}

\section{Ion Line Spectra (scanning cycle 3) on 15/08/2007}
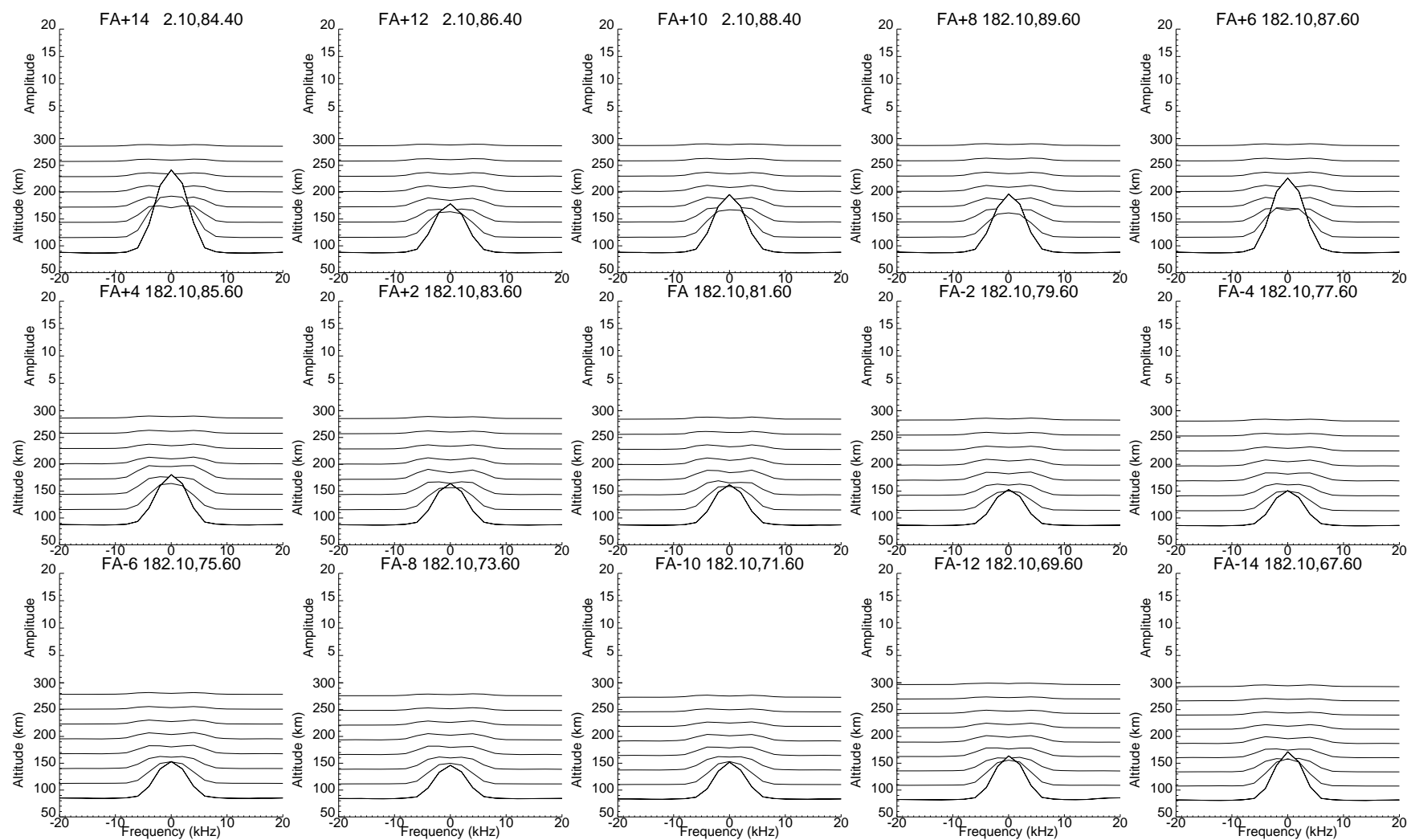

Fig. 10. Ion line spectra from scanning cycle 3 from interval 3. Since the cycles shown here comprise fifteen pointing directions instead of seven, the panel layout has been amended appropriately. Therefore fifteen panels have been used, one for each of the pointing directions from FA+14 to FA-14 (left to right and downwards), with the axes in each data panel again showing the frequency, the altitude and the spectral amplitude. As before, each panel direction is labelled using its designation, e.g. FA+10, followed by the azimuth and elevation of that pointing direction. The main features in this data are the characteristic E-region amplitude increases. These occur in many directions, from FA+14 to FA-6, with a notable prevalence of high-amplitude scatter being present around vertical. Although F-region spectral signatures are present, there is little evidence for SPEAR-induced amplitude increases in these F-region spectra.

There is evidence of an E-region throughout the three cycles, but this appears to be unaffected by the SPEAR high-power wave. The data in Fig. 9 contain fewer examples of F-region enhancements, although they do appear to show definite enhancements in the E-region, with these increases occurring in FA+10, FA+5, FA and FA-5.

We conclude our presentation of the data by considering the ion line spectra from interval 3. Although we previously highlighted scanning cycles 1, 3, 4 and 5 (Fig. 5), only ion line spectra from scanning cycle 3 have been presented. This is because enhanced plasma lines were seen during this cycle, which is consistent with these features being SPEARinduced. Additionally, cycle 3 contains the clearest examples of similar signatures that are also present in cycles 4 and 5. Furthermore, we have neglected to comment further on data from cycle 1 because of the dropout caused by the airport interlock. The data are shown in Fig. 10 and since cy- cle 3 comprises fifteen pointing directions instead of seven, the panel layout has been changed accordingly. Figure 10 consists of fifteen panels, one for each of the pointing directions from FA+14 to FA-14 (left to right and downwards), with the panel axes corresponding to the same parameters as before. The main features are clearly the characteristic Eregion amplitude increases that occur for a number of directions. These occur in many directions, from FA+14 to FA-6, with a prevalence of high-amplitude scatter being present around vertical. Although F-region spectral signatures are present, there is little evidence for SPEAR-induced amplitude increases in these F-region spectra. 


\section{Discussion}

In this study, we have provided ESR observations of the temporal evolution of aspect sensitive SPEAR-induced E- and F-region ion and plasma line spectral enhancements from the polar ionosphere over Svalbard. We have presented evidence of multi-directional spectral enhancements during three intervals when the SPEAR high-power facility was transmitting continuously in $\mathrm{O}$-mode at $4.45 \mathrm{MHz}$ while pointing field-aligned. We note the presence of SPEAR-enhanced incoherent backscatter from a variety of directions throughout the angular range $\left( \pm 15^{\circ}\right.$ centred on field-aligned) over which we have obtained our observations. These results illustrate clearly the dependence of RF-induced phenomena on their direction relative to the geomagnetic field. Where possible, ion line data were complemented by plasma line data, as upshifted and downshifted plasma line enhancements at the SPEAR pump frequency $( \pm 4.45 \mathrm{MHz}$ from the ESR transmitter frequency) were likely to be SPEAR-induced. On the other hand, (E-region $0 \mathrm{kHz}$ ) ion line enhancements can result from natural plasma density increases and such variability in the plasma density is indicative of the SPEAR-affected region of ionosphere being traversed by patches of overdense plasma (Robinson et al., 2006; Dhillon et al., 2007).

Ion line data from interval 1 (7 December 2005) include E-region amplitude enhancements in the central ion line and F-region amplitude enhancements in both the ionacoustic peaks and the central part of the ion line spectrum. SPEAR-enhanced plasma lines were also observed. For the porous sporadic E-layer in this interval, clear from Fig. 1, the E-region enhancements indicate that the transmitted pump wave energy excited electrostatic waves in the Eregion. This is consistent with patchy E-region plasma that is in frequent motion during SPEAR-on (Dhillon et al., 2007). It is also indicative of highly variable density-dependent absorption within and penetration through the sporadic E-layer during the SPEAR-on period. As mentioned by Robinson et al. (2006), the variability in the plasma density leads to strong local fluctuations in the electric field strength of the highpower wave. Therefore, the threshold electric fields necessary for instability excitation will only be exceeded intermittently and for short periods during the SPEAR-on interval. If the plasma density of the sporadic E-layer is high, then this implies that the majority of the high-power wave energy ought to be absorbed or reflected in the E-region, with little to propagate to the F-region. However, any RF energy that does manage to penetrate the patchy sporadic E-layer is free to propagate to the F-region, where, for sufficiently high F-region plasma density, it can excite the instabilities that enhance the F-region spectra. Previous SPEAR-induced spectral enhancements during the presence of a sporadic Elayer (Dhillon et al., 2007) have been attributed to the effects of cavitons (Djuth and Gonzales, 1988) and the observations discussed here may also be caused by the same physical process, further details of which are given below.
As for the data from interval 1, the E-region ion line spectra from interval 3 (15 August 2007) have a single central peak, indicating collisional plasma, rather than a pair of ionacoustic peaks and RF-induced phenomena caused an increase in the amplitude of this central peak. However, the ionograms from this interval (Fig. 3) do not indicate the presence of a sporadic E-layer during the SPEAR-on period. We therefore propose that these observations may be explained by invoking the PDI or the PGM (see below), which may also account for the E-region results reported by Rietveld et al. (2002). Although there is a degree of variability in the ion and plasma line data, there is some evidence to suggest that these ion line enhancements were accompanied by plasma line enhancements for pointing directions close to vertical. This is consistent with previous SPEAR-induced ion and plasma line enhancements, which have also tended to occur simultaneously (Robinson et al., 2006; Dhillon et al., 2007). As for the E-region data from interval 3, F-region ion line enhancements from interval 2 (9 October 2006), which occur in the central part of the ion line spectrum and in the ionacoustic peaks, also suggest that both the PDI and the PGM were operating, with F-region ionogram features (Fig. 2) indicating that the high-power wave interacted in the F-region.

Our results were obtained while SPEAR operated with an ERP of approximately $15 \mathrm{MW}$ and are consistent with a negligible SPEAR-induced change in the plasma temperature. As suggested by Robinson et al. (2006), this relatively low SPEAR pump power may result in limited heating of the plasma when compared to the temperature enhancements that are routinely seen using the EISCAT (Troms $\varnothing$ ) heater, which has an ERP of several hundred MW (Rietveld et al., 1993). Also, this negligible plasma heating implies a lack of interaction altitude descent driven by RF-induced reductions in the recombination rate. Such descent has been noted by Djuth et al. (1994) for vertical observations and those obtained from $5^{\circ}$ south of vertical, and by Dhillon and Robinson (2005) for field-aligned observations. This temperature-dependent effect has since been modelled by Ashrafi et al. (2006) whose results agreed with the findings of Djuth et al. (1994) and Dhillon and Robinson (2005), both of whose studies were undertaken using the EISCAT heater and mainland incoherent scatter radar system.

Turning now to the CUTLASS coherent backscatter data (Fig. 6) from interval 2 (9 October 2006), we see prominent SPEAR-induced backscatter enhancements observed by both the CUTLASS Finland (beam 9) and Iceland (beam 6) radars. SPEAR-enhanced CUTLASS backscatter, consistent with artificial field-aligned irregularities, and SPEAR-enhanced ESR backscatter have previously been observed simultaneously (Robinson et al., 2006; Dhillon et al., 2007). As noted by Robinson et al. (2006), such observations are in marked contrast to those typically obtained at Troms $\varnothing$, where irregularity onset, associated with upper-hybrid waves excited at the upper-hybrid resonance layer, is usually assumed to inhibit excitation of Langmuir and ion-acoustic waves, which 
occur near the O-mode reflection height, by redirecting the pump wave energy towards stimulating these upper-hybrid waves. Such simultaneous observations of coherent and incoherent backscatter indicate important differences between RF-induced effects in the ionospheres over Troms $\varnothing$ and Svalbard, and further investigations involving comparison of the backscatter amplitudes may yield important information regarding RF-induced instability processes in the polar ionosphere. We report similar simultaneous enhancements in this study, where, particularly between 12:00 and 15:00 UT (scanning cycles 4 to 14 in interval 2), variable ion line amplitudes are present together with artificial backscatter (Fig. 6). Combining these observations with the well accepted result that irregularities are generated at the upperhybrid height, which is close to where the O-mode pump wave is reflected, the ESR observations indicate that the artificial irregularities detected by CUTLASS were generated in the F-region, from where previous observations have been made and from where ray-tracing studies indicate that such scatter should originate (Robinson et al., 2006; Yeoman et al., 2007, 2008). These findings complement the study by Dhillon et al. (2007) who observed irregularities from upper E-/lower F-region altitudes. Further evidence that the scatter originated from the upper E-/lower F-region, and not from higher altitudes, was provided by Senior et al. (2004) who, following on from previous studies undertaken by Jones et al. (1984) and Robinson (1989), investigated the altitudinal extent of RF-induced artificial field-aligned irregularities and determined that they had e-folding scale lengths of approximately $20 \mathrm{~km}$.

As noted above, the observation of SPEAR-enhanced CUTLASS backscatter is highly susceptible to changes in the ionospheric plasma density, which affects the characteristics of the $1 \frac{1}{1 / 2}$-hop propagation path that is required to observe the scatter (Yeoman et al., 2007, 2008). Also, the variability in ionospheric conditions over extended periods affects the ability of the ionosphere to sustain artificial irregularities, e.g. if the plasma becomes underdense then artificial field-aligned irregularities will not be created. These factors combined would result in variable backscatter powers being measured by CUTLASS. Dhillon and Robinson (2005) proposed a relationship between field-aligned irregularities and routinely observed field-aligned RF-enhanced incoherent scatter, whereby artificially excited ion-acoustic and Langmuir waves could be guided preferentially along the geomagnetic field. This process may rely upon the irregularities, which are elongated and extend along the field lines, acting as waveguides and focusing along the geomagnetic field those waves whose wave vectors are generally parallel to it, thereby channelling RF-excited electrostatic waves in the field-aligned direction. This mechanism is consistent with our F-region ESR observations, where field-aligned SPEARexcited electrostatic waves were often observed. In addition, we have also seen repeated E-region field-aligned amplitude increases.
We now discuss the reported aspect sensitivity of various RF-induced features. Such aspect sensitive responses include those that have been observed in Langmuir turbulence (Isham et al., 1999), RF-induced descent of the interaction altitude (Djuth et al., 1994; Dhillon and Robinson, 2005), optical emissions (Kosch et al., 2000; Pedersen et al., 2003; Kosch et al., 2004), electron temperature increases (Rietveld et al., 2003) and stimulated electromagnetic emissions (SEE) (Isham et al., 2005). The field-aligned direction was found to be important in some of these studies, e.g. Rietveld et al. (2003) found the highest electron temperature increases in this direction. This agreed with the findings of Dhillon and Robinson (2005) whose observations of field-aligned RF-induced descent of the interaction altitude were explained using these high electron temperatures. Also, observations of artificially induced optical emissions (airglow) were also noted to occur preferentially in the fieldaligned direction (Kosch et al., 2000; Pedersen et al., 2003). However, other directions to the geomagnetic field have also given interesting results. For instance, RF-induced descent of the interaction altitude was noted by Djuth et al. (1994) in the vertical direction and from $5^{\circ}$ south of vertical. Also, Isham et al. (1999) found that RF-induced Langmuir turbulence maximized between field-aligned and the direction corresponding to the Spitze angle, which demarcates the Spitze region through which propagating high-power waves may reach their highest possible reflection point and excite instabilities that include the PDI and PGM. In addition, Dhillon and Robinson (2005) found persistent heater-enhanced incoherent backscatter from $6^{\circ}$ south of field-aligned. Such multi-directional backscatter was also recorded by Kosch et al. (2004), together with optical emissions, and the directions from which these heater-induced phenomena originated were found to vary over time. Furthermore, Isham et al. (2005) obtained evidence for the aspect sensitivity of different features present in SEE spectra.

Previous studies in which aspect sensitive phenomena have been reported have noted the importance of the Spitze region (e.g. Isham et al., 1999; Kosch et al., 2000), in which the angle of the pump waves to the vertical is less than the Spitze angle. Such high-power rays can reach the altitude where the pump frequency equals the local cold plasma frequency, thereby propagating through the PDI and PGM excitation region, which extends from the O-mode reflection height down to where Landau damping of the electrostatic waves becomes prohibitively large (Rietveld et al., 1993; Kohl et al., 1993). Importantly, Mishin et al. (2004, 2005) argued that heating outside the Spitze region, including in the field-aligned direction, was not conducive to the generation of Langmuir and ion-acoustic waves via the actions of the PDI and PGM. Consequently, Mishin et al. $(2004,2005)$ proposed an alternative mechanism for excitation of Langmuir and ion-acoustic waves outside the Spitze region. This process involved parametric decay of the O-mode pump wave into upper-hybrid (and lower-hybrid) waves. Together, these 
provide us with two mechanisms that could result in SPEARenhanced incoherent backscatter, depending upon whether the Spitze angle is exceeded by the propagating high-power waves.

We turn now to our observations and note that the Spitze angle at Svalbard is about $4^{\circ}$. From this we determine that, for data intervals 1 and 2, FA+5 and FA+10 lie within the Spitze region with FA+15 being just outside. Similarly for interval $3, \mathrm{FA}+12, \mathrm{FA}+10, \mathrm{FA}+8$ and $\mathrm{FA}+6$ are within the Spitze region, with FA+14, FA+4 and FA+2 being just outside. Taking into account previously recorded fieldaligned enhancements (e.g. Dhillon and Robinson, 2005), and those occurring between field-aligned and the Spitze angle (Isham et al., 1999), we may consider FA, FA+2, $\mathrm{FA}+4$ and the Spitze region directions given above as antenna orientations with which we may reasonably expect to see SPEAR-enhanced scatter. Although our observations indicate spectral enhancements from many directions, we note that SPEAR-enhanced incoherent backscatter was seen frequently in FA and in directions close to vertical. Field-aligned SPEAR-induced enhancements similar to those shown here have been observed previously (Robinson et al., 2006; Dhillon et al., 2007) and have been attributed to the actions of the PDI and PGM, although this appears to be inconsistent with the conditions described by Mishin et al. (2004, 2005). However, the results from close to vertical, recorded using pointing directions within the Spitze region, may be explained by invoking the PDI and PGM. Also, enhancements in FA+15 and FA+14 are very close to the edge of the Spitze region and may also be accounted for using the PDI and PGM. Perhaps more puzzling are the signatures from directions south of field-aligned. Such signatures were seen in FA-15 and repeatedly in FA-5. These observations in FA -5 are similar to the Troms $\varnothing$ results obtained by Dhillon and Robinson (2005) where ion line amplitude increases were seen from $6^{\circ}$ south of field-aligned. They are also in accordance with the findings of Ogawa and Oyama (private communications, 2007) who noted RFenhanced scatter from $6-8^{\circ}$ south of field-aligned at Troms $\varnothing$. As a final remark on aspect sensitivity, we suggest that our observations of enhanced scatter at FA-15 may indicate the presence of an ionospheric tilt, perhaps similar to that noted by Rietveld et al. (2003), with sufficient power being transmitted into the ionosphere due to the presence of a side lobe, as modelling of the beam pattern shows that the side lobes have maxima that occur at about $15^{\circ}$ off the centre of the main lobe.

We now discuss the effects of a high-power pump wave interacting with the kind of sporadic E-layer that was present during interval 1. Both Gordon and Carlson (1976) and Djuth (1984) observed sporadic E-layer plasma line enhancements exactly at the radar frequency \pm the pump frequency. Djuth (1984) concluded that the PGM was probably below threshold during these observations, and therefore direct conversion may play a role in explaining the sporadic E-layer data. Djuth and Gonzales (1988) subsequently examined the temporal development of the RF-enhanced sporadic E-layer plasma line in great detail and concluded that, although direct conversion of the pump wave into Langmuir waves by in situ small-scale irregularities can explain rapid (less than $20 \mu$ s) RF-enhanced plasma line growth, slower (greater than $100 \mu \mathrm{s}$ ) observed growth times are difficult to explain with this process. Instead, it was proposed that mode conversion along sporadic E-layer vertical gradients near the critical layer provides a better overall description of the observations, and that the formation of density cavities (cavitons) near the reflection height may play an essential role in the production of Langmuir waves. These cavitons (e.g. Morales and Lee, 1977) could give rise to the SPEAR-induced sporadic E-layer ion and plasma line enhancements reported here and similar ion line enhancements presented by Dhillon et al. (2007). Such cavitons and the spatial extent of associated density inhomogeneities are consistent with the study by Robinson (2002) who applied a multiple scatter theory to the propagation of electromagnetic test waves during RFinduced heating and found a broadening of the interaction region.

Furthermore, observed plasma line growth times in the range $<4$ to $20 \mu \mathrm{s}$, being much shorter than those anticipated for a convective PDI, led Newman et al. (1998) to suggest that an absolute (i.e. non-convective) PDI (Rosenbluth et al., 1973) could develop in the sporadic E-layer plasma. The absolute instability in an inhomogeneous plasma, whose threshold is much higher than that for the convective instability (e.g. Goldman et al., 1997), is not limited by convective losses, where the three-wave interaction matching condition that characterizes the convective instability is essentially detuned as Langmuir waves propagate along the steep electron density gradient (e.g. Bernhardt et al., 2003). This was used to explain the large sporadic E-layer airglow enhancements observed by Djuth et al. (1999) at altitudes of approximately $120 \mathrm{~km}$. Bernhardt et al. (2003) indicated that linear mode conversion may also be important in explaining the sporadic E-layer airglow. Also, Gondarenko et al. (2003) performed simulations that indicate that linear mode conversion in sporadic E-layer plasma above Arecibo gives rise to localized regions containing amplified electric fields. These enhanced electric fields could accelerate electrons and lead to the production of the intense airglow.

As stated above, SPEAR-induced E-region enhancements from interval 3 were collected during a period when the corresponding ionograms indicated that a sporadic E-layer was not present. This implies that, as for our F-region observations, the PDI and PGM may have been excited, as these results were probably obtained from a plasma regime similar to that studied by Rietveld et al. (2002), who presented ion and plasma line observations in an auroral E-layer having a scale height of $5-10 \mathrm{~km}$. This is significantly different from sporadic E-layer plasma, where the scale height is $0.2-1 \mathrm{~km}$ or less (Djuth, 1984). One expects the (convective) PDI and the 
PGM to be excited in plasma with a scale height of 5-10 km, but not in sporadic E-layer plasma where the electron density gradients are much steeper (Djuth, 1984). The reason for this is that in a steep sporadic E-layer electron density gradient, the Langmuir wave vector quickly rotates away from the direction of the pump field thereby decoupling from the pump. In addition, the wave rapidly propagates outside of the narrowly confined altitude region of instability preventing significant Langmuir wave amplification from occurring (e.g. Perkins and Flick, 1971; Fejer and Leer, 1972; Muldrew, 1978). Consequently, the thresholds of the PGM and the standard convective PDI become extremely large and therefore the PDI and PGM do not represent viable processes with which to explain the production of Langmuir waves in sporadic E-layers. This argument is supported by theoretical studies of Langmuir turbulence in collisional E-region conditions undertaken by Goldman et al. $(1995,1997)$.

\section{Conclusions}

We have presented an aspect sensitivity study of SPEARinduced ESR spectral enhancements from the E- and Fregions of the polar ionosphere. As alluded to above, the ionosphere over Svalbard is highly variable with patches of overdense plasma that drift in and out of the region of influence of SPEAR and field-of-view of the ESR. This indicates that the thresholds for instability excitation are exceeded only intermittently during SPEAR-on. This high level of ionospheric variability therefore leads to inconsistent observations of SPEAR-induced backscatter. Furthermore, such variability affects the repeatability of the experiment, since the ionosphere may have changed while cycling through the set of directions, so that notable enhancements in a particular direction in one scanning cycle may not be present in that direction during subsequent scanning cycles. Therefore, we have applied several scanning cycles in order to test the aspect sensitive response of the ionosphere to the high-power pump wave and to examine whether this response provides consistent signatures in any pointing direction. We have observed sporadic E-layer enhancements indicating the presence of cavitons and E- and F-region enhancements consistent with the actions of both the PGM and the PDI. We have noted features from a variety of directions, some of which were frequently observed in field-aligned, vertical and south of field-aligned. Reasons for the prevalence of observations from (close to) vertical include the existence of the Spitze region. In addition, observations of enhanced field-aligned scatter may be aided by the presence of field-aligned irregularities, which may guide the electrostatic waves that are excited by RF-induced instabilities. However, evidence appears to be growing that the ionospheric region corresponding to pointing directions of about $5-8^{\circ}$ south of field aligned is worthy of further investigation, and our Svalbard observations of RF-enhanced incoherent backscatter from $5^{\circ}$ south of field-aligned provide such contributory evidence. Collectively these findings indicate the significance of the directional dependence of RF-induced phenomena and highlight the importance of further investigating the aspect sensitivity of such effects. Finally, we should remark that these results highlight the value of plasma physical experiments conducted in the polar cap ionosphere using the SPEAR highpower facility, which satisfactorily complement previous observations from lower latitudes.

Acknowledgements. The SPEAR facility is supported on grant PPA/Z/O/2002/00668 by the UK Particle Physics and Astronomy Research Council (PPARC). CUTLASS is supported on PPARC grant PPA/R/R/1997/00256, and by the Finnish Meteorological Institute and the Swedish Institute of Space Physics. Many thanks are due to the EISCAT Scientific Association for ESR operations and support. The authors would also like to thank L. Baddeley, S. Imber, L. Clausen and the technical staff for valuable support during the experimental campaigns.

Topical Editor K. Kauristie thanks M. Kosch and another anonymous referee for their help in evaluating this paper.

\section{References}

Ashrafi, M., Kosch, M. J., and Honary, F.: Heater-induced altitude descent of the EISCAT UHF ion line enhancements: observations and modelling, Adv. Space Res., 38, 2645-2652, 2006.

Bernhardt, P. A., Gondarenko, N. A., Gudzar, P. N., Djuth, F. T., Tepley, C. A., Sulzer, M. P., Ossakow, S. L., and Newman, D. L.: Using radio-induced aurora to measure the horizontal structure of ion layers in the lower thermosphere, J. Geophys. Res., 108, 1336, doi:10.1029/2002JA009712, 2003.

Carlson, H. C. and Duncan, L. M.: HF excited instabilities in space plasmas, Radio Sci., 12, 1001-1013, 1977.

Carlson, H. C., Gordon, W. E., and Showen, R. L.: High frequency induced enhancements of the incoherent scatter spectrum, J. Geophys. Res., 77, 1242-1250, 1972.

Clausen, L. B. N., Yeoman, T. K., Wright, D. M., Robinson, T. R., Dhillon, R. S., and Gane, S. C.: First results of a ULF wave injected on open field lines by SPEAR, J. Geophys. Res., 113, A01305, doi:10.1029/2007JA012617, 2008

Dhillon, R. S., Robinson, T. R., and Wright, D. M.: Radar $\mathrm{ACF}$ and turbulence characteristics from artificially generated field-aligned irregularities, Geophys. Res. Lett., 29, 1830, doi:10.1029/2002GL015364, 2002.

Dhillon, R. S. and Robinson, T. R.: Observations of time dependence and aspect sensitivity of regions of enhanced UHF backscatter associated with RF heating, Ann. Geophys., 23, 7585,2005 ,

http://www.ann-geophys.net/23/75/2005/.

Dhillon, R. S., Robinson, T. R., and Yeoman, T. K.: EISCAT Svalbard radar observations of SPEAR-induced E- and F-region spectral enhancements in the polar cap ionosphere, Ann. Geophys., 25, 1801-1814, 2007, http://www.ann-geophys.net/25/1801/2007/.

Djuth, F. T.: HF-enhanced plasma lines in the lower ionosphere, Radio Sci., 19, 383-394, 1984. 
Djuth, F. T. and Gonzalez, C. A.: Temporal evolution of the HFenhanced plasma line in sporadic E, J. Geophys. Res., 93, 196208, 1988

Djuth, F. T., Stubbe, P., Sulzer, M. P., Kohl, H., Rietveld, M. T., and Elder, J. H.: Altitude characteristics of plasma turbulence excited with the Troms $\varnothing$ superheater, J. Geophys. Res., 99, 333 339, 1994.

Djuth, F. T., Bernhardt, P. A., Tepley, C. A., Gardner, J. A., Kelley, M. C., Broadfoot, A. L., Kagan, L. M., Sulzer, M. P., Elder, J. H., Selcher, C., Isham, B., Brown, C., and Carlson, H. C.: Large airglow enhancements produced via wave-plasma interactions in sporadic E, Geophys. Res. Lett., 26, 1557-1560, 1999.

Fejer, J. A.: Ionospheric modification and parametric instabilities, Rev. Geophys., 17, 135-153, 1979.

Fejer, J. A. and Leer, E.: Purely growing parametric instability in an inhomogeneous plasma, J. Geophys. Res., 77, 700-708, 1972.

Goldman, M. V., Newman, D. L., Russell, D., DuBois, D. F., Rose, H., Drake, R. P., and Rubenchik, A. M.: Collisional regimes of radiation-driven Langmuir turbulence, Phys. Plasmas, 2, 19471960, 1995.

Goldman, M. V., Newman, D. L., Drake, R. P., and Afeyan, B. B.: Theory of convective saturation of Langmuir waves during ionospheric modification of a barium cloud, J. Atmos. Sol. Terr. Phys., 59, 2335-2350, 1997.

Gondarenko, N. A., Gudzar, P. N., Ossakow, S. L., and Bernhardt, P. A.: Linear mode conversion in inhomogeneous magnetized plasmas during ionospheric modification by HF radio waves, J. Geophys. Res., 108, 1470, doi:10.1029/2003JA009985, 2003.

Gordon, W. E. and Carlson, H. C.: Arecibo heating experiments, Radio Sci., 9, 1041-1047, 1974.

Gordon, W. E. and Carlson, H. C.: The excitation of plasma lines in blanketing sporadic E, J. Geophys. Res., 81, 4016-4018, 1976.

Honary, F., Robinson, T. R., Wright, D. M., Stocker, A. J., Rietveld, M. T. and McCrea, I.: First direct observations of the reduced striations at pump frequencies close to the electron gyroharmonics, Ann. Geophys., 17, 1235-1238, 1999, http://www.ann-geophys.net/17/1235/1999/.

Isham, B., Rietveld, M. T., Hagfors, T., La Hoz, C., Mishin, E., Kofman, W., Leyser, T. B., and van Eyken, A. P.: Aspect angle dependence of HF enhanced incoherent backscatter, Adv. Space Res., 24, 1003-1006, 1999.

Isham, B., Hagfors, T., Khudukon, B., Yurik, R. Yu., Tereshchenko, E. D., Rietveld, M. T., Belyey, V., Grill, M., La Hoz, C., Brekke, A., and Heinselman, C.: An interferometer experiment to explore the aspect angle dependence of stimulated electromagnetic emission spectra, Ann. Geophys., 23, 55-74, 2005, http://www.ann-geophys.net/23/55/2005/.

Jones, T. B., Robinson, T. R., Stubbe, P., and Kopka, H.: Frequency dependence of anomalous absorption caused by high power radio waves, J. Atmos. Terr. Phys., 46, 147-153, 1984.

Kagan, L. M., Kelley, M. C., Garcia, F., Bernhardt, P. A., Djuth, F. T., Sulzer, M. P. and Tepley, C. A.: The structure of electromagnetic wave-induced 557.7-nm emission associated with a sporadic-E event over Arecibo, Phys. Rev. Lett., 85, 218-221, 2000.

Kagan, L. M., Bakhmet'eva, N. V., Belikovich, V. V., Tolmacheva, A. V., and Kelley, M. C.: Structure and dynamics of sporadic layers of ionization in the ionospheric E region, Radio Sci., 37, 1106, doi:10.1029/2001RS002534, 2002.
Kohl, H., Kopka, H., Stubbe, P., and Rietveld, M. T.: Introduction to ionospheric heating at Troms $\varnothing$-II. Scientific problems, J. Atmos. Terr. Phys., 55, 601-613, 1993.

Kosch, M. J., Rietveld, M. T., Hagfors, T., and Leyser, T. B.: Highlatitude HF-induced airglow displaced equatorward of the pump beam, Geophys. Res. Lett., 27, 2817-2820, 2000.

Kosch, M. J., Rietveld, M. T., Senior, A., McCrea. I. W., Kavanagh, A. J., Isham, B., and Honary, F.: Novel artificial optical annular structures in the high latitude ionosphere over EISCAT, Geophys. Res. Lett., 31, L12805, doi:10.1029/2004GL019713, 2004.

Milan, S. E., Yeoman, T. K., Lester, M., Thomas, E. C., and Jones, T. B.: Initial backscatter occurrence statistics from the CUTLASS HF radars, Ann. Geophys., 15, 703-718, 1997, http://www.ann-geophys.net/15/703/1997/.

Mishin, E. V., Burke, W. J., and Pedersen, T.: On the onset of HF-induced airglow at HAARP, J. Geophys. Res., 109, A02305, doi:10.1029/2003JA010205, 2004.

Mishin, E. V., Burke, W. J., and Pedersen, T.: HF-induced airglow at magnetic zenith: theoretical considerations, Ann. Geophys., 23, 47-53, 2005,

http://www.ann-geophys.net/23/47/2005/.

Morales, G. J. and Lee, Y. C.: Generation of density cavities and localized electric fields in a nonuniform plasma, Phys. Fluids, 20, 1135-1147, 1977.

Muldrew, D. B.: Langmuir wave propagation and the enhanced plasma line in sporadic E, J. Geophys. Res., 83, 5207-5211, 1978.

Newman, D. L., Goldman, M. V., Djuth, F. T., and Bernhardt, P. A.: Langmuir turbulence associated with ionospheric modification: Challenges associated with recent observations during a sporadic-E event, Physics of Space Plasmas, vol. 15, edited by: Chang, T. and Jasperse, J. R., 259-264, Mass. Inst. of Technol., 1998.

Pedersen, T. R., McCarrick, M., Gerken, E., Selcher, C., Sentman, D., Carlson, H. C., and Gurevich, A.: Magnetic zenith enhancement of HF radio-induced airglow production at HAARP, Geophys. Res. Lett., 30, 1169, doi:10.1029/2002GL016096, 2003.

Perkins, F. W. and Flick, J.: Parametric instabilities in inhomogeneous plasmas, Phys. Fluids, 14, 2012-2018, 1971.

Perkins, F. W. and Kaw, P. W.: On the role of plasma instabilities in ionospheric heating by radio waves, J. Geophys. Res., 76, 282284, 1971.

Rietveld, M. T., Kohl, H., Kopka, H., and Stubbe, P.: Introduction to ionospheric heating at Troms $\varnothing-$ I. Experimental overview, J. Atmos. Terr. Phys., 55, 577-599, 1993.

Rietveld, M. T., Isham, B., Kohl, H., La Hoz, C., and Hagfors, T.: Measurements of HF-enhanced plasma and ion lines at EISCAT with high-altitude resolution, J. Geophys. Res., 105, 7429-7439, 2000.

Rietveld, M. T., Isham, B., Grydeland, T., La Hoz, C., Leyser, T. B., Honary, F., Ueda, H., Kosch, M., and Hagfors, T.: HFpump-induced parametric instabilities in the auroral E-region, Adv. Space. Res., 29, 1363-1368, 2002.

Rietveld, M. T., Kosch, M. J., Blagoveshchenskaya, N. F., Kornienko, V. A., Leyser, T. B., and Yeoman, T. K.: Ionospheric electron heating, optical emissions, and striations induced by powerful HF radio waves at high latitudes: aspect angle dependence, J. Geophys.. Res., 108, 1141, doi:10.1029/2002JA009543, 2003. 
Robinson, T. R.: The heating of the high latitude ionosphere by high power radio waves, Phys. Rep., 179, 79-209, 1989.

Robinson, T. R.: Effects of multiple scatter on the propagation and absorption of electromagnetic waves in a field-aligned-striated cold magneto-plasma: implications for ionospheric modification experiments, Ann. Geophys., 20, 41-55, 2002, http://www.ann-geophys.net/20/41/2002/.

Robinson, T. R., Yeoman, T. K., Dhillon, R. S., Lester, M., Thomas, E. C., Thornhill, J. D., Wright, D. M., van Eyken, A. P., and McCrea, I. W.: First observations of SPEAR induced artificial backscatter from CUTLASS and the EISCAT Svalbard radars, Ann. Geophys., 24, 291-309, 2006, http://www.ann-geophys.net/24/291/2006/.

Rosenbluth, M. N., White, R. B., and Liu, C. S.: Temporal evolution of a three-wave parametric instability, Phys. Rev. Lett., 31, 11901193, 1973.

Schlegel, K., Rietveld, M., and Maul, A.: A modification event of the auroral E region as studied with EISCAT and other diagnostics, Radio. Sci., 22, 1063-1072, 1987.

Scoffield, H. C., Yeoman, T. K., Robinson, T. R., Baddeley, L. J., Dhillon, R. S., Wright, D. M., Raita, T., and Turunen, T.: First results of artificial stimulation of the ionospheric Alfvén resonator at $78^{\circ} \mathrm{N}$, Geophys. Res. Lett., 33, L19103, doi:10.1029/2006GL027384, 2006.

Senior, A., Borisov, N. D., Kosch, M. J., Yeoman, T. K., Honary, F., and Rietveld, M. T.: Multi-frequency HF radar measurements of artificial F-region field-aligned irregularities, Ann. Geophys., 22, 3503-3511, 2004, http://www.ann-geophys.net/22/3503/2004/.
Stubbe, P., Kohl, H., and Rietveld, M. T.: Langmuir turbulence and ionospheric modification, J. Geophys. Res., 97, 6285-6297, 1992.

Stubbe, P.: Review of ionospheric modification experiments at Tromsø, J. Atmos. Terr. Phys., 58, 349-368, 1996.

Wannberg, G., Wolf, I., Vanhainen, L.-G., Koskenniemi, K., Röttger, J., Postila, M., Markkanen, J., Jacobsen, R., Stenberg, A., Larsen, R., Eliassen, S., Heck, S., and Huuskonen, A.: The EISCAT Svalbard radar: a case study in modern incoherent scatter radar system design, Radio Sci., 32, 2283-2307, doi:10.1029/97RS01803, 1997.

Wright, D. M., Davies, J. A., Robinson, T. R., Chapman, P. J., Yeoman, T. K., Thomas, E. C., Lester, M., Cowley, S. W. H., Stocker, A. J., Horne, R. B., and Honary, F.: Space Plasma Exploration by Active Radar (SPEAR): an overview of a future facility, Ann. Geophys., 18, 1248-1255, 2000, http://www.ann-geophys.net/18/1248/2000/.

Yeoman, T. K., Blagoveshchenskaya, N., Kornienko, V., Robinson, T. R., Dhillon, R. S., Wright, D. M., and Baddeley, L. J.: SPEAR: early results from a very high latitude ionospheric heating facility, Adv. Space Res., 40, 384-389, 2007.

Yeoman, T. K., Chisham, G., Baddeley, L. J., Dhillon, R. S., Karhunen, T. J. T., Robinson, T. R., Senior, A., and Wright, D. M.: Mapping ionospheric backscatter measured by the SuperDARN HF radars - Part 2: Assessing SuperDARN virtual height models, Ann. Geophys., 26, 843-852, 2008, http://www.ann-geophys.net/26/843/2008/. 